\title{
Identidad profesional docente en la formación universitaria: una revisión sistemática de estudios cualitativos
}

\author{
David Cuadra-Martínez ${ }^{\star *}$, Pablo J. Castro-Carrasco ${ }^{2,3}$, Cristian Oyanadel ${ }^{4,5}$ e Ingrid N. González-Palta ${ }^{2,6}$ \\ (1) Facultad de Humanidades y Educación, Dpto. de Psicología, Univ. de Atacama, Atacama-Chile. \\ (correo-e: david.cuadra@uda.cl) \\ (2) Facultad de Humanidades, Dpto. de Psicología, Univ. de La Serena, Coquimbo-Chile. \\ (correo-e: pablocastro@userena.cl; igonzalez@userena.cl) \\ (3) Universidad Católica del Maule, Maule-Chile. \\ (4) Facultad de Ciencias Sociales, Dpto. de Psicología, Univ. de Concepción, Concepción-Chile. \\ (correo-e: coyanadel@udec.cl) \\ (5) Centro de Investigación Mente, Cerebro y Comportamiento, Grupo CTS-261, Univ. de Granada, Granada-España. \\ (6) Facultad de Psicología, Univ. Alberto Hurtado, Santiago-Chile.
}

* Autor a quien debe ser dirigida la correspondencia

Recibido Ene. 5, 2021; Aceptado Mar. 8, 2021; Versión final Abr. 8, 2021, Publicado Ago. 2021

\begin{abstract}
Resumen
El objetivo de este trabajo fue sistematizar la información de estudios cualitativos sobre el desarrollo de la identidad profesional (IP) del profesor(a) durante la formación inicial. La IP del profesor(a) es una variable clave para comprender la vida profesional de los profesores(as), siendo la formación inicial un período especialmente crítico para su desarrollo. Se realizó una revisión sistemática de la literatura científica, utilizando las bases de datos WOS (Web of Science), Scopus y Scielo desde el año 2000 al 2020. Se encontraron 67 artículos, incluyéndose 33 en el análisis cualitativo y cuantitativo, luego de filtrar según criterios de inclusión y exclusión. Se encontró un conjunto de categorías que favorecen el desarrollo de la IP, un proceso de construcción conflictuado entre las dimensiones subjetivas y objetivas de la IP, además de dimensiones que constituyen la IP. Se concluye que el desarrollo de la IP del docente se ve influenciado por la formación inicial, las características psicológicas y los factores socioculturales.
\end{abstract}

\section{Teacher's professional identity during university education: a systematic review of qualitative research}

\begin{abstract}
The main objective of this study is to perform a systematic review of qualitative research on the development of teacher's professional identity $(\mathrm{PI})$ during their initial training. Teacher's $\mathrm{PI}$ is a key variable in understanding the professional life of teachers, with initial training being an especially critical period for their development. A systematic review of the scientific literature published on WOS (Web of Science), Scopus, and Scielo databases is performed from the years 2000 to 2020. Sixty-seven articles are retrieved, of which 33 are selected for qualitative and quantitative analyses after filtering by using inclusion and exclusion criteria. The results show a set of categories that favor the development of PI. This is a developing process in which there are conflicts between subjective and objective dimensions, as well as other dimensions that comprise PI. It is concluded that teacher PI development is affected by initial training, by psychological characteristics, and by socio-cultural factors.
\end{abstract}

Keywords: professional identity; teacher; subjectivity; initial training; qualitative research 


\section{INTRODUCCIÓN}

La identidad profesional del profesor(a), en adelante IP, concentra el interés de numerosos investigadores(as) en todo el mundo, considerándose un factor clave para comprender la vida profesional de los profesores(as). A la fecha, se sabe que un mayor desarrollo de ésta promueve un mejor bienestar y desempeño docente, genera altos niveles de compromiso con la profesión, además de un mayor rendimiento académico en los estudiantes. La formación inicial docente es un período especialmente crítico para la construcción de la IP. Es durante este período que los estudiantes deben transformarse en profesores(as), lo que implica desarrollar una serie de competencias que en el futuro, les permitan desempeñar la profesión docente en un contexto que a menudo desafía la calidad de la formación recibida, impone una serie de exigencias y dificultades que los futuros docentes deben superar, además de exigir calidad en los resultados obtenidos en términos de aprendizaje. Por lo anterior, se requiere comprender mayormente cómo se desarrolla la IP docente durante este período, cómo es que se produce este proceso y qué efectos produce sobre la IP desarrollada.

La IP se ha considerado una construcción integrada por una dimensión subjetiva, ya que representa la forma en que las personas se ven a sí mismas con respecto a una profesión, incluyendo un conjunto de saberes, sentimientos y emociones para autodefinirse como tal, que emergen a partir de la interacción social (Bolívar et al., 2014). Desde esta perspectiva, el sistema de significados que las personas construyen sobre la profesión, es lo que permite identificarse como profesor(a). En este proceso, considerar las experiencias vividas por el estudiante durante su formación inicial y el ejercicio de la profesión, es fundamental para comprender la construcción de esta identidad y para que los estudiantes se identifiquen como docentes.

Dado que actualmente hay un cuerpo de literatura científica de estudios empíricos que describen qué promueve el desarrollo de la IP del profesor(a), cómo es el proceso de construcción de esta identidad y cuáles son los efectos de este desarrollo, se torna pertinente y relevante sistematizar la información que durante algunas décadas los investigadores(as) de la IP docente han reportado. Este trabajo tiene como objetivo sistematizar la información de estudios empíricos sobre el desarrollo de la IP del docente durante la formación inicial, que han buscado comprender este constructo desde una dimensión subjetiva y desde los principales actores de este proceso.

El concepto de identidad es difícil de definir, existiendo múltiples acepciones de acuerdo a la disciplina que lo aborde. Sin embargo, en términos generales se puede decir que este concepto se refiere a cómo las personas responden a la pregunta de quiénes son. Mientras que la IP se caracteriza por ser multidimensional e integrar la identidad personal. Para comprenderla, es necesario considerarla como un proceso y un producto. En cuanto al producto, se define como el conjunto de significados que la persona se atribuye como profesional, a partir de la interacción de sus características personales con las propias de la profesión. Como proceso, implica una transformación sucesiva de la forma en que la persona se define como profesional, a partir de factores sociales, culturales, contextuales y personales. Además, la IP es dinámica, intersubjetiva y se encuentra integrada por subidentidades que en ocasiones entran en conflicto. A partir de la IP, los docentes establecen una misión profesional, desarrollan atributos característicos de su profesión, valores, creencias, un conjunto de saberes, además de autoeficacia profesional (Zhu, 2017). De esta manera, un mayor desarrollo de la IP docente lo agencia para el mejoramiento educativo, lo orienta en la toma de decisiones profesionales, produciendo un mayor compromiso con la profesión y un mejor desempeño profesional.

La interacción social, especialmente con los actores relacionados con la profesión de profesor(a), es lo que de manera sucesiva permite la construcción del significado y sentido subjetivo de la profesión, en donde se integran el cuerpo normativo regulatorio propio de la profesión docente, además de las costumbres gremiales y comportamientos culturales de los profesores(as). Uno de los contextos sociales de mayor influencia en el desarrollo de la IP del docente, es la formación universitaria, por lo que es necesario aportar evidencias científicas que permitan comprender este proceso de construcción durante la formación inicial (van der Wal et al., 2019). Las características de los programas de estudio en la formación docente tienen un papel central en la construcción de la IP. Cuando se forma a los futuros profesores(as), esta tarea se lleva a cabo consciente o inconscientemente o de manera planificada o no, de acuerdo con algún(os) modelo(s) de formación docente, que en sus fundamentos ontológicos y epistemológicos, asume lo que significa ser un profesor(a).

Desde la clasificación de los modelos formativos docentes de Tezgiden (2016), el modelo tecnicista asume que los profesores(as) mantienen un rol profesional pasivo y una enseñanza que consiste en la transmisión de conocimientos elaborados por otros especialistas en el área. Una formación inicial docente basada en este modelo, se centrará predominantemente en la entrega de saberes disciplinarios a los estudiantes de pedagogía e imprimirá en los futuros profesores(as), una imagen de profesional meramente ejecutor y transmisor de conocimientos. Por otro lado, un modelo hermenéutico reflexivo identifica al profesorado como un profesional reflexivo, que toma decisiones en base a estas reflexiones, y por lo tanto, es autónomo y generador del conocimiento que enseña. Actualmente este modelo formativo es el que la literatura científica 
fomenta para formar profesores(as) e implica gestar en ellos un rol profesional proactivo en el ejercicio de la profesión. Finalmente, desde un modelo crítico, el profesor(a) es una figura intelectual y transformadora de la sociedad, que lucha frente a las presiones ideológicas e injusticias sociales que tensionan el trabajo docente. Una formación inicial fundamentada en este modelo, podría desarrollar una IP en los futuros profesores(as), caracterizada por la crítica social y el desarrollo de ésta en sus estudiantes, además de la responsabilidad de producir los cambios sociales.

En una línea similar, Beijaard (2019) ha planteado que tradicionalmente a los docentes se los identifica como expertos en la materia, expertos en la didáctica o expertos en la pedagogía. Como experto en la materia, se lo valora por el dominio del conocimiento que enseña, siendo menos importante la forma en que lo enseña. Mientras que como didacta, al profesor(a) se lo identifica como un experto en estrategias para generar aprendizajes. Como experto pedagogo, el docente es identificado como un profesional que apoya el desarrollo integral del estudiante, siendo una competencia clave la ética para resolver los cada vez más frecuentes problemas morales que surgen en los contextos educativos.

Desde una mirada más específica, la formación universitaria impacta en la IP de los estudiantes de pedagogía, a partir del conocimiento que el estudiante adquiere de la profesión y las relaciones interpersonales que surgen en este contexto, en donde cobra especial valor la interacción con los formadores de profesores(as) y cuánto se reconoce en ellos la IP docente. Beijaard (2019), en una misma línea, posiciona a la reflexión sobre el proceso de profesionalización docente, la interacción con el contexto y el desarrollo de la agencia en los futuros profesores, como promotores de mayores niveles de IP. Un especial lugar ocupan los programas formativos que fomentan en sus estudiantes el asumir roles y responsabilidades profesionales en los contextos educativos reales - prácticas tempranas - dado que esta estrategia permite la construcción de la IP. En esta misma línea, los académicos(as) y profesores(as) mentores ocupan un rol relevante, siendo el trabajo colaborativo entre estudiantes, académicos(as) y profesionales asociados al campo de la educación, una de las estrategias generadoras de IP docente (Nguyen y Loughland, 2018).

Comprender la complejidad del desarrollo de la IP docente, implica considerar una variedad de formas de investigar este proceso. Para Cardoso et al. (2014), la investigación cualitativa permite acceder a la dimensión emocional y experiencial de la IP del profesor(a), develar el proceso de esta construcción y el significado o sentido que se otorga a la profesión y desde allí, identificar qué elementos de la formación inicial impactan en esta construcción. La dimensión subjetiva de la IP del profesor(a) se integra por las experiencias, emociones y conocimientos subjetivos, con los cuáles se otorga un significado y sentido a la información y conocimientos formales u objetivados que emergen en los contextos formativos o se instalan como representaciones sociales de lo que significa en determinada cultura ser docente. De hecho, para Živković (2018), construir IP docente implica conciliar la vida personal con la vida profesional, lo cual produce tensiones que podrían llevar incluso al abandono de la carrera profesional.

Estas tensiones entre las experiencias, expectativas y emociones - subjetividad - con el conocimiento formal y normativo propio de la profesión, concluye con un mayor desarrollo de la IP docente cuando se logra la integración de estas dimensiones (Hendrikx, 2020). Complementando lo anterior, Beijaard (2019) ha reportado evidencia de que para el desarrollo sistemático de la IP del docente se requiere más que nunca de la dimensión subjetiva, dado que los profesores(as) necesitan asumir un rol de pedagogos cuya principal función es tomar decisiones éticas frente a los problemas morales. Frente a los dilemas morales que los maestros enfrentan en los contextos educativos, es preciso que reflexionen e incorporen aspectos éticos, experienciales y emocionales para orientar sus prácticas pedagógicas. Desde esta perspectiva, es necesario que a los docentes se los forme para lograr integrar los conocimientos subjetivos, profesionales y científicos, porque desde allí es que podrán sustentar la toma de decisiones profesionales. Dada la importancia de la subjetividad del docente en el desarrollo de la IP, se torna pertinente y relevante conocer qué hallazgos reportan los estudios cualitativos que han considerado la dimensión subjetiva de la IP durante la formación inicial.

\section{METODOLOGÍA}

Corresponde a una revisión sistemática de la literatura científica. Para esto, se utilizó la definición de revisión sistemática y estructura propuesta por el método de Cochrane. Se analizaron documentos científicos en la base de datos Wos, Scopus y Scielo, desde el año 2000 al 2020, utilizando los siguientes criterios de inclusión: (a) investigaciones empíricas cualitativas o que incluyan resultados cualitativos; (b) escritas en español e inglés; (c) que trate sobre la IP docente en la formación inicial o que incluya resultados al respecto; (d) la muestra sobre la que se realice el estudio se encuentre en un proceso de formación pre-servicio profesional. Se excluyó (a) literatura gris (tesis, congresos, libros) dada la dificultad de acceso a esta información y la posible menor calidad del proceso de evaluación científica que caracteriza a esta literatura, (b) y documentos en donde no fue posible identificar claramente la metodología utilizada. El motor de búsqueda incluyó 
"investigación cualitativa + identidad profesional + estudiante de pedagogía o profesor en formación o formación docente" y en inglés qualitative research + professional identity + pre-service teacher or qualitative research + professional identity + preservice teacher. La búsqueda se estableció en base al título del artículo, el abstract y/o las palabras clave. Se encontraron 67 artículos, sometiendo finalmente 33 a análisis luego de filtrar en base a los criterios de inclusión y exclusión, como lo muestra la figura 1 . El análisis se realizó cualitativamente en base a análisis de contenido y cuantitativamente, en base a frecuencias, en base a una matriz que consideró (a) los elementos que favorecen el desarrollo de la IP; (b) el proceso de desarrollo de la IP; (c) la IP como producto y (d) las características metodológicas de los estudios revisados. La Tabla 1 muestra los manuscritos revisados.
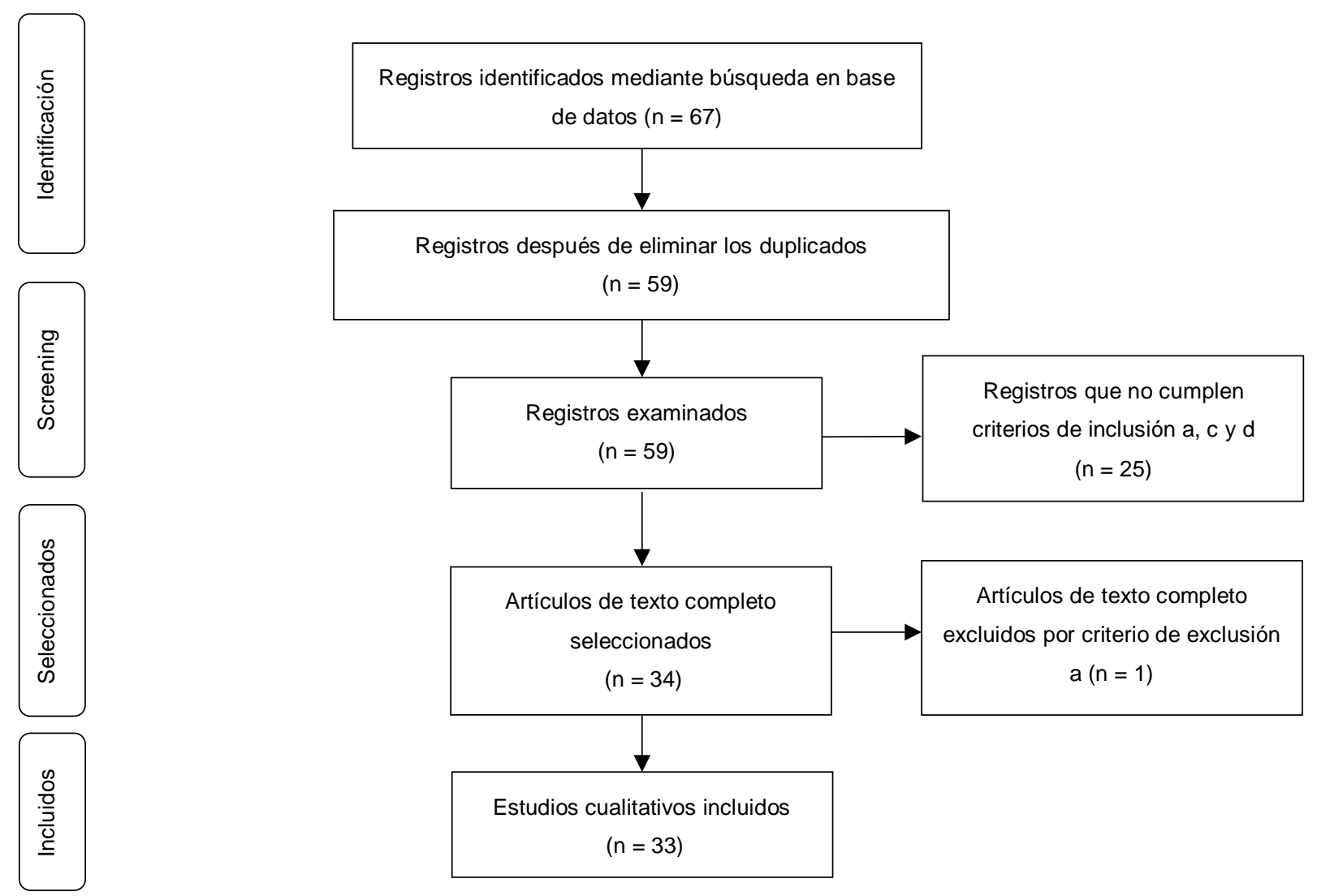

Fig. 1: Proceso de búsqueda y selección de los artículos revisados.

Tabla 1: Artículos revisados

\begin{tabular}{|c|c|c|c|c|}
\hline$N^{\circ}$ & País/región & Año & Autor & Participantes \\
\hline 1 & \multirow[t]{2}{*}{ Sudáfrica } & 2020 & Chikoko, R., y Msibi, T. & $\begin{array}{l}6 \text { estudiantes de Pedagogía en Educación Parvularia, que cursan } \\
\text { su último año }\end{array}$ \\
\hline 2 & & 2018 & $\begin{array}{l}\text { Steenekamp, K., van der Merwe, M., y } \\
\text { Mehmedova, A.S. }\end{array}$ & 137 estudiantes de pedagogía \\
\hline 3 & México & 2020 & Flores, L., Olave, I., y Villarreal, A. & $\begin{array}{l}15 \text { estudiantes de pedagogía en inglés del noveno semestre, en } \\
\text { proceso de práctica profesional }\end{array}$ \\
\hline 4 & \multirow[t]{3}{*}{ Australia } & 2019 & Arnold, J. & 1 estudiante de pedagogía de 4to año \\
\hline 5 & & 2016 & Rourke, A., y Snepvangers, K. & 62 estudiantes de pedagogía para la enseñanza el arte \\
\hline 6 & & 2019 & Gray, C., Wright, P., y Pascoe, R. & 18 estudiantes de pedagogía en teatro, en práctica profesional \\
\hline 7 & \multirow[t]{2}{*}{ Chile } & 2019 & Alvarado, M., Neira, M., y Westmacott, A. & 12 estudiantes de pedagogía en inglés que cursan su último año \\
\hline 8 & & 2019 & Barahona, M., y lbaceta, X. & 8 estudiantes de pedagogía en inglés \\
\hline
\end{tabular}


Tabla 1: continuación

\begin{tabular}{|c|c|c|c|c|}
\hline$N^{\circ}$ & País/región & Año & Autor & Participantes \\
\hline 9 & \multirow[t]{7}{*}{ EEUU } & 2018 & Hubbard, J. & $\begin{array}{l}5 \text { estudiantes de pedagogía en educación primaria que } \\
\text { participaron de un curso formativo de estudios sociales que } \\
\text { incluyó un proceso de práctica en un colegio }\end{array}$ \\
\hline 10 & & 2014 & McGee, E. & $\begin{array}{l}13 \text { estudiantes de pedagogía en educación primaria con } \\
\text { especialidad en matemática }\end{array}$ \\
\hline 11 & & 2011 & Katz, P., McGinnis, R., y otros cinco autores & $\begin{array}{l}25 \text { estudiantes de pedagogía, especialidad de ciencias, de } \\
\text { distintos niveles educativos }\end{array}$ \\
\hline 12 & & 2000 & Newman, C. & 39 estudiantes de pedagogía de primer y último año \\
\hline 13 & & 2020 & Kerkhoff, S., Broere, M., y Premont, D. & 19 estudiantes de pedagogía \\
\hline 14 & & 2017 & $\begin{array}{l}\text { Carrier, S., Whitehead, A., y otros tres } \\
\text { autores }\end{array}$ & 16 estudiantes de pedagogía de 1er año \\
\hline 15 & & 2000 & Broaddus, $\mathrm{K}$ & $\begin{array}{l}1 \text { estudiante de 4to año de un programa de licenciatura en } \\
\text { artes y una maestría en educación }\end{array}$ \\
\hline 16 & Canadá & 2019 & Moore, S. & $\begin{array}{l}5 \text { estudiantes de pedagogía, que asisten a un curso de } \\
\text { aprendizaje de una segunda lengua }\end{array}$ \\
\hline 17 & Vietnam & 2019 & Le, L.T., y Dwyer, L.G. & $\begin{array}{l}19 \text { estudiantes de pedagogía en inglés que cursan 3er y 4to } \\
\text { año de su carrera, además de formadores de profesores }\end{array}$ \\
\hline 18 & \multirow[t]{4}{*}{ Turquía } & 2015 & Sakallı-Gümüş, S. & $\begin{array}{l}32 \text { estudiantes de pedagogía en educación especial cursando } \\
\text { una práctica temprana }\end{array}$ \\
\hline 19 & & 2019 & Köksal, D., y Genç, G. & $\begin{array}{l}8 \text { estudiantes de pedagogía que cursan } 4 \text { to año y participan de } \\
\text { un programa intensivo para enseñanza de inglés }\end{array}$ \\
\hline 20 & & 2011 & Özmen, K.S. & 67 estudiantes de pedagogía en inglés \\
\hline 21 & & 2020 & Dikilitaş, K., y Comoglu, I. & 53 estudiantes de pedagogía inglés de primer año \\
\hline 22 & Etiopía & 2019 & Ayetenew, A. & $\begin{array}{l}\text { Estudiantes de pedagogía, empleadores, documentos políticos } \\
\text { sobre formación docente, formadores de profesores }\end{array}$ \\
\hline 23 & \multirow[t]{3}{*}{ Hong-Kong } & 2018 & Trent, J. & $\begin{array}{l}6 \text { Estudiantes de pedagogía en Licenciatura en educación, con } \\
\text { especialización en inglés, que realizan práctica profesional, } \\
\text { además de formadores de profesores }\end{array}$ \\
\hline 24 & & 2018 & Trent, J. & Egresados de pedagogía en inglés \\
\hline 25 & & 2013 & Trent, J., y Shroff, R. & $\begin{array}{l}6 \text { estudiantes de pedagogía en inglés, que cursan 3er año y se } \\
\text { encuentra en práctica }\end{array}$ \\
\hline 26 & \multirow{3}{*}{ Brasil } & 2018 & Machado, A., y Rodrigues de Mattos, C.R. & 52 estudiantes de pedagogía en física \\
\hline 27 & & 2015 & Teixeira, B.R., y Cyrino, M.C. & 10 estudiantes de pedagogía en matemáticas, de 3ro y 4to año \\
\hline 28 & & 2017 & Oliveira, $\mathrm{H}$. & $\begin{array}{l}5 \text { estudiantes de pedagogía en lenguas o letras portuguesa, } \\
\text { becarios de un programa }\end{array}$ \\
\hline 29 & China & 2017 & Zhao, H., y Zhang, X. & $\begin{array}{l}12 \text { estudiantes pedagogía que cursan práctica profesional, de } \\
\text { distintas especialidades (química, inglés, literatura, física, } \\
\text { tecnología y educación especial) }\end{array}$ \\
\hline 30 & \multirow[t]{2}{*}{ Inglaterra } & 2016 & Bradshaw, P., y Younie, S. & $\begin{array}{l}18 \text { estudiantes de pedagogía que participaron en un programa } \\
\text { escolar sobre noticias }\end{array}$ \\
\hline 31 & & 2015 & Woolhouse, C. y Cochrane, M. & 159 estudiantes de pedagogía en matemáticas y ciencias \\
\hline 32 & Irlanda & 2015 & Rutherford, V., Conway, P., y Murphy, R. & $\begin{array}{l}17 \text { estudiantes que cursan un postgrado para calificar para la } \\
\text { docencia, además de tutores y mentores de práctica }\end{array}$ \\
\hline 33 & Venezuela & 2008 & $\begin{array}{l}\text { Sayago, Q., Zoraida, B., Chacón, M., y } \\
\text { Rojas de Rojas, M. }\end{array}$ & 4 estudiantes de pedagogía en educación básica integral \\
\hline
\end{tabular}

\section{RESULTADOS}

El análisis de los estudios cualitativos revisados permitió describir la identidad profesional del docente, a partir de cuatro grandes categorías que dan cuenta de los factores asociados al desarrollo de esta identidad, el proceso de construcción de la misma, el resultado de esta construcción y las características de las metodologías de los estudios utilizados.

\section{Desarrollo de la identidad profesional del docente en la formación inicial}

Se encontró un conjunto de factores en los estudios cualitativos revisados, que interactúan e inciden en el desarrollo de la IP en los estudiantes de pedagogía. Estos factores se pueden clasificar en aquellos asociados al (a) programa de estudio, (b) las características psicológicas de los estudiantes y (c) los factores socioculturales. 


\section{El programa de estudio}

Tres dimensiones destacan como parte de los programas de estudio de formación docente, que son generadores de IP. El primero de éstos, es el enfoque formativo del programa de estudio. Específicamente, es el énfasis en formar a los estudiantes de pedagogía en base a una integración teórica práctica, el aprendizaje autónomo, la reflexión y el trabajo en equipo o aprendizaje colaborativo, lo que promueve el desarrollo de la IP docente. También se encontraron iniciativas que apuntan a nivelar o reforzar los conocimientos disciplinares y pedagógicos, además de actividades de enseñanza diseñadas específicamente para favorecer la IP de los estudiantes.

Las figuras del profesor(a) mentor (docente supervisor del centro de práctica profesional) y del tutor (académico que guía la práctica profesional) son especialmente relevantes, existiendo ciertas condiciones y características de éstos, que pueden favorecer o no el desarrollo de la IP del docente. De igual forma, los procesos de práctica son una de las experiencias formativas que más se asocia al desarrollo de la IP. Destacan como elementos incidentes en la IP, las prácticas iniciadas tempranamente, una adecuada articulación universidad-escuela, una relación de apoyo y cercanía de los profesores(as) mentores y tutores, el fomento de la autonomía y el liderazgo en los estudiantes practicantes, además de experiencias de aprendizaje desafiantes. La Tabla 2 detalla estos resultados.

Tabla 2: Características de los programas de estudio y desarrollo de la IP

\begin{tabular}{|c|c|c|}
\hline Elementos & Descripción & Artículo $N^{\circ}$ \\
\hline \multirow[t]{6}{*}{$\begin{array}{l}\text { Enfoque } \\
\text { formativo } \\
\text { del } \\
\text { programa } \\
\text { de estudio }\end{array}$} & $\begin{array}{l}\text { Énfasis en superar la dualidad teoría-práctica, en base a experiencias tempranas } \\
\text { de inserción de los estudiantes en contextos laborales: destacan las prácticas } \\
\text { tempranas, las pasantías a colegios, el trabajo en red con organismos o } \\
\text { instituciones ligadas a la educación }\end{array}$ & $\begin{array}{l}2-5-8-9-11-18-19- \\
23-26-27-28-30\end{array}$ \\
\hline & Promoción de la autonomía y el aprendizaje autónomo & 31 \\
\hline & $\begin{array}{l}\text { Énfasis en la formación de estudiantes reflexivos, en base a didácticas o } \\
\text { estrategias planificadas para la reflexión que permitan integrar los saberes } \\
\text { subjetivos-biográficos, el saber disciplinar y técnico adquirido en la universidad, } \\
\text { además del conocimiento obtenido en la práctica. }\end{array}$ & $1-2-5-7-19-28$ \\
\hline & $\begin{array}{l}\text { Énfasis en la formación en trabajo en equipo o aprendizaje colaborativo, } \\
\text { incluyendo académicos y representantes del campo profesional en estos grupos }\end{array}$ & $\begin{array}{l}2-5-7-8-18-19-28- \\
30-31\end{array}$ \\
\hline & $\begin{array}{l}\text { Oferta de programas de nivelación o profundización en conocimientos } \\
\text { disciplinarios y/o pedagógicos (en ciencia, en arte para planificar didácticas, en } \\
\text { didácticas, entre otros) }\end{array}$ & $11-14-19-20-31$ \\
\hline & $\begin{array}{l}\text { Oferta de actividades pedagógicas, asignaturas diseñadas para desarrollar IP o } \\
\text { actividades extracurriculares. Por ejemplo, lectura de historias profesionales de } \\
\text { docentes, unidades de estudio sobre IP, asignatura sobre IP y actividades de } \\
\text { voluntariado para enseñar a grupos comunitarios }\end{array}$ & $8-13-21-33$ \\
\hline \multirow[t]{8}{*}{$\begin{array}{l}\text { La figura } \\
\text { del tutor } y \\
\text { del mentor }\end{array}$} & $\begin{array}{l}\text { La observación del desempeño de los docentes mentores durante procesos de } \\
\text { práctica, el asumir el rol de ayudante del mentor y la retroalimentación por parte } \\
\text { del mentor, favorecen el desarrollo de la IP }\end{array}$ & 3 \\
\hline & $\begin{array}{l}\text { Mentores hábiles, que hacen sentir al estudiante como profesional se } \\
\text { transforman en modelos profesionales a seguir. Específicamente, son } \\
\text { inspiradores, amplían los lazos profesionales con otros docentes (insertan en } \\
\text { nuevos contextos) y tienen buena relación con los estudiantes }\end{array}$ & $6-8$ \\
\hline & $\begin{array}{l}\text { Un mentor percibido como "mal profesional", puede impactar desarrollando un } \\
\text { bajo sentido de autoeficacia en los estudiantes en práctica o bien, alentarlos a } \\
\text { aprender a no replicar las prácticas pedagógicas negativas }\end{array}$ & 3 \\
\hline & $\begin{array}{l}\text { La poca asignación de tiempo de un mentor para supervisar prácticas, también } \\
\text { podría agenciar a los estudiantes en práctica para el autoaprendizaje. }\end{array}$ & 26 \\
\hline & $\begin{array}{l}\text { Un trato profesional simétrico y de ayuda del mentor con el practicante favorece } \\
\text { la IP }\end{array}$ & $3-4-29$ \\
\hline & $\begin{array}{l}\text { El apoyo del mentor para la adaptación del estudiante en práctica en la escuela } \\
\text { favorece la IP }\end{array}$ & 23 \\
\hline & Un supervisor que apoya, asesora, anima y evalúa, promueve la IP & $23-6$ \\
\hline & $\begin{array}{l}\text { Mentores que son ex estudiantes de la universidad, generan mayor confianza en } \\
\text { los practicantes para probar diferentes técnicas de enseñanza, mayor sentido de } \\
\text { colegialidad y más receptividad a la retroalimentación }\end{array}$ & 30 \\
\hline
\end{tabular}


Tabla 2: continuación

\begin{tabular}{|l|l|l|}
\hline Elementos & \multicolumn{1}{|c|}{ Descripción } & \multicolumn{1}{|c|}{ Artículo $N^{\circ}$} \\
\hline $\begin{array}{l}\text { La práctica } \\
\text { en contexto } \\
\text { real }\end{array}$ & $\begin{array}{l}\text { Favorecer el liderazgo y la autonomía del practicante al interior de la escuela, } \\
\text { promueve la IP }\end{array}$ & $4-5-30-8$ \\
\cline { 2 - 3 } & Animar a los practicantes a asumir riesgos desarrolla la IP & 30 \\
\cline { 2 - 3 } & $\begin{array}{l}\text { A menudo, un proceso de práctica produce inicialmente inseguridad y temor en } \\
\text { los practicantes }\end{array}$ & 18 \\
\cline { 2 - 3 } & $\begin{array}{l}\text { La práctica profesional podría generar sobrecarga y estrés en los practicantes, } \\
\text { situación que tensiona la IP }\end{array}$ & 26 \\
\cline { 2 - 3 } & $\begin{array}{l}\text { Los conflictos/contradicción/incidentes/problemas/casos durante la práctica } \\
\text { profesional, agencian en la construcción de la IP o bien obstaculizan la } \\
\text { identificación con la profesión, lo que dependerá del apoyo otorgado al } \\
\text { practicante }\end{array}$ & $26-5-18-15$ \\
\cline { 2 - 3 } & $\begin{array}{l}\text { El desajuste de las expectativas sobre el rol profesional docente transmitido en la } \\
\text { universidad, respecto del que se ejerce en las escuelas, puede conllevar al } \\
\text { abandono escolar/profesional }\end{array}$ & 23 \\
\cline { 2 - 3 } & $\begin{array}{l}\text { Herramientas que permitan al estudiante organizar el material de estudio, los } \\
\text { documentos de trabajo, las evidencias de sus logros de aprendizaje (por ejemplo } \\
\text { un portafolio), facilitan la construcción de la IP }\end{array}$ & 25 \\
\cline { 2 - 3 } & $\begin{array}{l}\text { La práctica concebida como una actividad universitaria dentro de una escuela, } \\
\text { dificulta el desarrollo de la IP, debido a que los practicantes se esfuerzan más } \\
\text { por cumplir las tareas universitarias que las escolares }\end{array}$ & 26 \\
\hline
\end{tabular}

\section{Características psicológicas de los estudiantes}

Un conjunto de características psicológicas de los estudiantes se asocian al desarrollo de la IP docente, que hemos agrupado en (a) capacidades cognitivas, (b) características de personalidad, (c) experiencias educativas/personales infantiles y (d) creencias acerca de la profesión. Para que los estudiantes desarrollen su IP, se requiere de capacidades cognitivas que le permitan avanzar en su profesionalización, es decir, lograr la adquisición de un conjunto de saberes que lo habilite para la profesión. En los estudios revisados, destacan la capacidad de observación, evaluación, reflexión, aprendizaje autónomo, creatividad e involucramiento académico (Flores et al., 2020; Rourke y Snepvangers, 2016).

Algunos estudios incluyen características de personalidad que el estudiante debe poseer o desarrollar, que se atribuyen a la identidad de docente, destacando la paciencia, amor, simpatía, compasión y prosocialidad (Chikoko y Msibi, 2020), además del comportamiento ético (Le y Dwyer, 2019). En cuanto a las experiencias de los estudiantes de pedagogía, se reportan experiencias infantiles educativas y personales de injusticia y carencias como promotoras de la motivación por ser profesor(a) para lograr cambios sociales (Arnold, 2019; Broaddus, 2000). También los vínculos afectivos y de apoyo establecidos con los docentes durante la infancia, promueven el modelado de su comportamiento (Chikoko y Msibi, 2020; Sayago et al., 2008). Por otra parte, aquellas experiencias infantiles que implican asumir roles de cuidado, protección y enseñanza a otros, motiva a ser docente (Arnold, 2019; McGee, 2014).

Finalmente, un cuerpo de evidencia devela que las creencias que los estudiantes tienen de la profesión, la enseñanza y el aprendizaje, pueden favorecer o no el desarrollo de la IP. Varios estudios revisados advierten de la importancia de promover el autoconocimiento de estas creencias, incluyendo las metas, expectativas e incluso los sueños asociados a la profesión (Alvarado et al., 2019; Carrier et al., 2017). Por ejemplo, Flores et al. (2020) señalan que elevadas expectativas de los estudiantes sobre el rol profesional de los docentes (un manejo perfecto de la disciplina, competencias pedagógicas extraordinarias, además de certificaciones de título destacables) podrían tensionar el desarrollo de la IP una vez que contrastan estas expectativas con el ejercicio de la profesión como estudiantes en práctica profesional.

\section{Factores socioculturales}

Algunos estudios revisados describen la influencia de los factores socioculturales en el desarrollo de la IP de los estudiantes de pedagogía. Las características de la relación entre las instituciones asociadas con la formación profesional y el estudiante de pedagogía, además de la información que este último recibe de parte de éstas sobre su desempeño, influye decididamente en la forma en que un estudiante construye su IP. La Tabla 3 describe lo anterior. 
Tabla 3: Factores socioculturales que inciden en la construcción de la IP

\begin{tabular}{|c|c|c|}
\hline Dimensión & Características & Art. $N^{\circ}$ \\
\hline \multirow[t]{2}{*}{$\begin{array}{l}\text { Relación } \\
\text { universidad/centro de } \\
\text { práctica }\end{array}$} & $\begin{array}{l}\text { Discrepancias entre las competencias formadas en educación superior y } \\
\text { las competencias requeridas en el trabajo, dificultan la construcción de la } \\
\text { IP }\end{array}$ & 22 \\
\hline & $\begin{array}{l}\text { Una vinculación institucionalizada entre la universidad y la escuela, que } \\
\text { genere condiciones adecuadas para la práctica, promueve el desarrollo de } \\
\text { la IP }\end{array}$ & 26 \\
\hline $\begin{array}{l}\text { Relación } \\
\text { universidad/organizaciones } \\
\text { ligadas a la profesión }\end{array}$ & $\begin{array}{l}\text { La inserción de estudiantes en actividades planificadas por organizaciones } \\
\text { ligadas a la educación favorece la IP }\end{array}$ & 9 \\
\hline \multirow[t]{3}{*}{$\begin{array}{l}\text { Representaciones sociales } \\
\text { de la profesión }\end{array}$} & $\begin{array}{l}\text { La representación social de la profesión y las estructuras de poder, } \\
\text { prescriben una imagen de profesor(a) al interior de la escuela que los } \\
\text { estudiantes en práctica deben cumplir. Destaca las formas prescritas de } \\
\text { comportarse y la presentación personal de un maestro }\end{array}$ & 32 \\
\hline & A menudo, al docente se lo identifica como un ejemplo de moral & 17 \\
\hline & $\begin{array}{l}\text { La infravaloración de la profesión docente puede desanimar la } \\
\text { identificación con la profesión }\end{array}$ & 26 \\
\hline \multirow[t]{2}{*}{ Estudiantes del practicante } & $\begin{array}{l}\text { El afecto y preocupación de los estudiantes de un practicante, promueven } \\
\text { el desarrollo de la IP }\end{array}$ & 29 \\
\hline & $\begin{array}{l}\text { El logro en términos de aprendizaje de los estudiantes de un practicante } \\
\text { fomenta el desarrollo de la IP }\end{array}$ & 12 \\
\hline \multirow{5}{*}{$\begin{array}{l}\text { Relación centro de } \\
\text { práctica/estudiante en } \\
\text { práctica }\end{array}$} & $\begin{array}{l}\text { La autonomía, liderazgo y reconocimiento del estatus profesional del } \\
\text { estudiante en práctica, promueve el desarrollo de la IP }\end{array}$ & $\begin{array}{l}4-12- \\
30\end{array}$ \\
\hline & Recibir retroalimentación de distintos agentes educativos promueve la IP & 12 \\
\hline & Recibir un trato respetuoso y de valoración promueve la IP & \\
\hline & $\begin{array}{l}\text { La inserción del estudiante en práctica en equipos de trabajo al interior de } \\
\text { la escuela, favorece el desarrollo de la IP }\end{array}$ & 30 \\
\hline & $\begin{array}{l}\text { Una cultura escolar centrada en los contenidos y no en el aprendizaje, } \\
\text { puede desanimar la identificación con la profesión }\end{array}$ & 26 \\
\hline $\begin{array}{l}\text { Relación universidad- } \\
\text { profesor en formación }\end{array}$ & $\begin{array}{l}\text { El reconocimiento del estudiante como un profesional en formación o } \\
\text { como un profesional especializado promueve la IP }\end{array}$ & $31-8$ \\
\hline $\begin{array}{l}\text { Relación estudiante de } \\
\text { pedagogía/medio social }\end{array}$ & $\begin{array}{l}\text { Interacciones positivas entre estudiantes de pedagogía y los símbolos, } \\
\text { recursos y personas relacionados con la profesión, favorecen el desarrollo } \\
\text { de la IP }\end{array}$ & 3 \\
\hline
\end{tabular}

\section{El proceso de construcción de la identidad profesional}

El análisis e interpretación de los estudios revisados, permite proponer un modelo heurístico en tres etapas del proceso de construcción de la IP en la formación inicial docente, que se grafica en la figura 2. La primera etapa corresponde a una pre IP docente, que se desarrolla principalmente en base a saberes intuitivos producto de las experiencias biográficas educativas y relacionadas con el rol docente, además de la representación social que culturalmente se tiene de los profesores(as) y la retroalimentación de los otros significantes de la profesión docente. Con esta pre IP los estudiantes ingresan a la formación universitaria, en base a tres grandes interrogantes que en lo sucesivo lo orientarán en el camino a recorrer para formarse como profesor(a). La primera y más importante pregunta en esta etapa, implica responder si realmente quiere ser profesor(a), lo que resuelve a partir de las fuentes de conocimientos ya señaladas anteriormente. La segunda y tercera pregunta implica responder si puede ser profesor(a) y cuánto siente que es un profesor(a), interrogantes que cobran mayor protagonismos en la medida que avanza en su formación profesional docente.

En el transcurso de los años formativos, el estudiante comienza a construir su IP principalmente en base a los conocimientos formales de tipos disciplinarios y técnicos. Representa un período de aprendizaje complejo y acelerado de lo que significa ser docente, pero principalmente en base a conocimientos disciplinarios y técnicos y mucho menos basados en la experiencia profesional: la pregunta relativa a si puede o no ser profesor(a) cobra mayor presencia en este período. La última etapa, que denominamos IP confirmada, se produce en los últimos años formativos y en base a la experiencia profesional en contextos laborales, desde donde integra principalmente conocimientos y experiencias propias del mundo del trabajo docente y además, forja su propia opinión de lo que significa ser profesor(a) y de cómo es como profesor(a). Todo este proceso implica el juicio crítico, la reflexión y el autoconocimiento a partir de estas tres grandes preguntas, cuyas respuestas incluyen emociones asociadas a la profesión de profesor(a), un sentido de autoeficacia, sentimientos de pertenencia a la profesión y la aceptación de los grupos sociales al gremio profesional. 

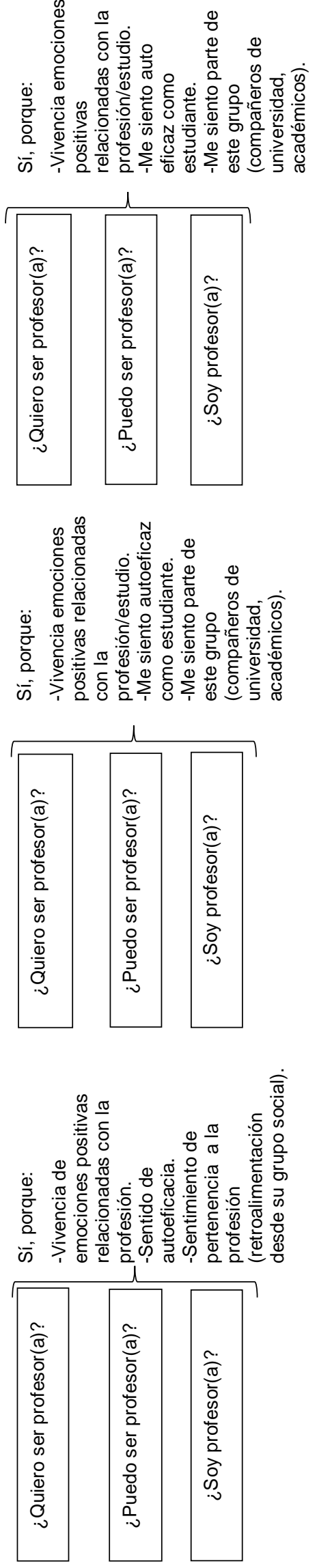
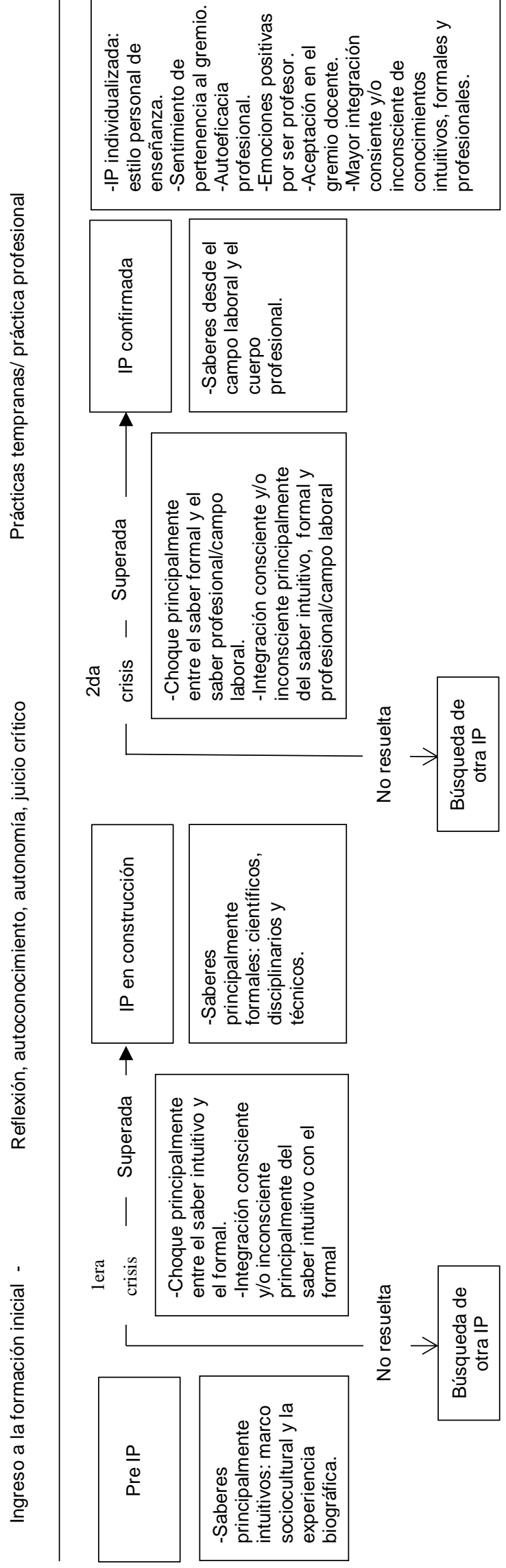

Fig. 2: Modelo heurístico del proceso de construcción de la IP en la formación inicial docente basado en los estudios revisados 


\section{Características de la identidad profesional desarrollada}

Los estudios revisados reportan que los estudiantes de pedagogía, en la medida que construyen una IP, adquieren (a) roles, (b) saberes, (c) características de personalidad, (d) tradiciones, (e) y desarrollan el sentimiento de ser parte de esta profesión. Este desarrollo de la IP considera particularidades de lo que significa culturalmente ser docente en cada sociedad, no obstante la Tabla 4 presenta las características esenciales de lo que significa ser profesor(a), a partir de los estudios revisados.

Tabla 4: Dimensiones del ser profesor

\begin{tabular}{|c|c|c|}
\hline Dimensión & Descripción & Artículo $N^{\circ}$ \\
\hline \multirow{5}{*}{ Roles } & $\begin{array}{l}\text { El docente debe amar a los estudiantes y la educación, además de } \\
\text { proteger, servir y conocer a los aprendices }\end{array}$ & $1-2-4-33$ \\
\hline & $\begin{array}{l}\text { El docente debe ser un ejemplo de moral para la comunidad y guía } \\
\text { espiritual de los estudiantes }\end{array}$ & 17 \\
\hline & $\begin{array}{l}\text { El docente debe ser un profesional crítico y reflexivo, resiliente, } \\
\text { comprometido y transformador de la sociedad }\end{array}$ & $2-21-8$ \\
\hline & $\begin{array}{l}\text { El docente debe enseñar y gestionar la clase; planificar la enseñanza y } \\
\text { cumplir roles para el funcionamiento de la organización escolar }\end{array}$ & 29-18-33 \\
\hline & El docente debe ser solidario, inspirador y un agente de cambio & 2 \\
\hline \multirow{10}{*}{ Saberes } & Competencias pedagógicas & 3-11-30-31-8 \\
\hline & $\begin{array}{l}\text { Saberes de especialidad disciplinar, pedagógico y técnicos (TIC, } \\
\text { /integración teoría práctica) }\end{array}$ & $9-20-30-31-27-18-25-33-28$ \\
\hline & Conocimiento de los aprendices & 11-16-18 \\
\hline & Conciencia de la importancia de la comunicación con los estudiantes & $29-30$ \\
\hline & Competencias para la formación moral & 17 \\
\hline & Conocimientos para el trabajo colaborativo & $19-30$ \\
\hline & Conciencia de que la enseñanza es una tarea compleja & $19-33-8$ \\
\hline & Conocimiento del campo laboral & $5-18-23-33-8-28$ \\
\hline & Conciencia del valor del trabajo colaborativo & $7-30-27$ \\
\hline & Conciencia de ser fundamental en la vida de los estudiantes & 4 \\
\hline \multirow{9}{*}{$\begin{array}{l}\text { Características } \\
\text { de } \\
\text { personalidad }\end{array}$} & Autoeficacia profesional & $\begin{array}{l}2-3-4-7-9-11-16-19-18-29- \\
30-31\end{array}$ \\
\hline & Liderazgo, trabajo en equipo y autonomía & $30-08-2028$ \\
\hline & Disposición al autoaprendizaje & jul-33 \\
\hline & Autoestima profesional & 07-sep \\
\hline & Juicio crítico del campo profesional & $3-23-27-28$ \\
\hline & Tolerancia y paciencia & 19 \\
\hline & Innovación y creatividad & 30-ago \\
\hline & Adaptación & 30 \\
\hline & Ético, intachable y correcto & 17 \\
\hline \multirow{3}{*}{$\begin{array}{l}\text { Costumbres y } \\
\text { tradiciones }\end{array}$} & $\begin{array}{l}\text { Reunirse en los recreos/tiempos no lectivos a compartir con los } \\
\text { colegas (sala de profesores(as) por ejemplo) }\end{array}$ & 19 \\
\hline & Aleccionar a los estudiantes en el patio & 19 \\
\hline & Adaptarse a los roles y costumbres de los docentes & 23 \\
\hline \multirow{6}{*}{$\begin{array}{l}\text { Pertenencia a } \\
\text { la profesión }\end{array}$} & Auto reconocimiento/confirmación de ser profesor(a) & $2-9-11-12-19-29-15-8-28$ \\
\hline & Reconocimiento de la profesión desde otros & 30 \\
\hline & Desarrollo de un estilo propio de enseñanza & $21-23$ \\
\hline & Motivación por ser profesor & $3-9-27-29$ \\
\hline & Sentimientos positivos hacia la profesión & $12-19-30-28$ \\
\hline & Pertenencia al grupo profesional & $30-31-33$ \\
\hline
\end{tabular}

\section{Metodologías utilizadas de los estudios revisados}

Los estudios cualitativos revisados reportan una amplia variedad de diseños para estudiar la IP docente durante la formación inicial. En la mayoría de los estudios se utilizó un diseño de estudio de casos, representando el 39\% de los estudios, mientras que el $9 \%$ utilizó más de un diseño y otro $9 \%$ se basó en un diseño longitudinal. En varias de las investigaciones no fue posible identificar el tipo de diseño empleado $(27 \%)$, mientras que en el $6 \%$ se utilizó un diseño de historias de vida, un $3 \%$ de investigación acción y un $3 \%$ etnográfico. En cuanto a la muestra, la mayoría de las investigaciones $(36 \%)$ se focalizó en estudiantes de los últimos años cursados, incluyendo cuarto año y el período de práctica profesional. El 15\% se focalizó en estudiantes de años iniciales (primer y segundo año), el 15\% incluyó estudiantes de varios niveles educativos, un $12 \%$ de un nivel intermedio (tercer año), un 3\% consideró a aquellos que recientemente habían egresado, mientras que en el $21 \%$ de los estudios no fue posible identificar el nivel cursado por los estudiantes de pedagogía. Finalmente, el 18\% consideró a docentes de escuelas (mentores) o académicos (formador de profesores/as) como parte de la muestra. 
Con respecto al procedimiento de recolección de información, se encontró una amplia variedad de instrumentos, considerando la mayoría de los estudios la incorporación de más de una herramienta de recolección de información. En el $58 \%$ de los estudios se utilizó algún tipo de entrevista. También destaca el uso de grupos focales, de discusión o talleres para recabar datos (27\%), al igual que los diarios reflexivos cuya función es que el estudiante de pedagogía registre por escrito sus experiencias y reflexiones sobre procesos formativos prácticos (27\%). Un $18 \%$ incluyó el análisis de documentos, por ejemplo, políticas educativas, trabajos o tareas realizadas por los estudiantes, el 15\% utilizó algún tipo de grabación del desempeño en una clase (audio o audiovisual), el 15\% utilizó observaciones cualitativas, el 12\% empleó alguna técnica gráfica (dibujos, pintura, carteles), mientras que un $6 \%$ se basó en cuestionarios de preguntas abiertas.

\section{DISCUSIÓN}

El objetivo de este trabajo fue sistematizar la información de los estudios cualitativos sobre el desarrollo de la IP docente durante la formación inicial. Comprender la dimensión subjetiva de la IP del profesor(a) es fundamental para lograr promover un modelo formativo que identifique al docente como un profesional reflexivo, capaz de integrar el saber subjetivo con el saber formal, para desde allí abordar los complejos problemas morales y socio ambientales que actualmente enfrenta la educación (Beijaard, 2019). Probablemente una de las dificultades de la formación inicial docente al desarrollar sistemáticamente la IP, es precisamente contar con los conocimientos y herramientas formativas que permitan la integración de la multidimensionalidad de la IP. Este trabajo aporta en algún nivel a lo anterior, en la medida que proporciona una sistematización de los hallazgos de estudios empíricos que han considerado a la IP del profesor(a) desde una dimensión subjetiva, lo que podría orientar los programas formativos en el abordaje de la IP docente.

La revisión de la literatura reporta que son tres elementos los que inciden en el desarrollo de la IP del docente durante la formación inicial. El primero de éstos, es el programa de estudio, situación ya advertida por Tezgiden (2016) y Beijaard (2019), quienes destacan la incidencia de los modelos formativos a la base de la formación inicial docente. Desde la revisión realizada, aquellos programas de estudio que se focalizan en la integración teórica práctica, el aprendizaje autónomo, la reflexión, el trabajo en equipo o aprendizaje colaborativo, la nivelación de competencias o actividades formativas especialmente diseñadas para desarrollar la IP, son generadores de mayores niveles de desarrollo de la IP docente. La reflexión ha sido considerada desde hace unas décadas una característica distintiva necesaria para la profesionalización docente (Beijaard, 2019) o adquisición de los saberes necesarios para el adecuado ejercicio de la profesión, por lo que ha orientado la formación docente a focalizarse en el aprendizaje reflexivo. Desde estos hallazgos, se requiere un adecuado complemento entre los procesos reflexivos y la integración teórica práctica, el aprendizaje colaborativo y sobre todo, actividades diseñadas específicamente para desarrollar IP, y para la mejora continua de la formación docente, tanto inicial como en la práctica.

Además, la figura del mentor y del académico son especialmente relevantes, pudiendo impulsar la identificación con la profesión o bien incluso promover el abandono de la carrera. Si bien la literatura advierte de la necesidad de desarrollar un aprendizaje y trabajo colaborativo entre estudiantes, académicos y mentores (Nguyen y Loughland, 2018), desde los hallazgos obtenidos también se debe considerar que tanto mentores como académicos deben ser agentes formativos que establezcan un vínculo de apoyo, confianza y respeto con los estudiantes, para que se produzca el desarrollo de la IP, para ellos necesitan ser formados en cómo aportar a una construcción reflexiva de la IP. Probablemente en la medida que éstos reúnan estas condiciones, será posible promover una efectiva comunidad de aprendizaje, que no solo promueva el desarrollo profesional del estudiante en práctica, sino que también de los docentes involucrados en la formación de profesores(as).

Sumado a lo anterior, el hallazgo que más reportan los estudios como generador de IP docente en los estudiantes, son los procesos de práctica profesional, advirtiendo de la importancia de iniciar estas actividades formativas lo antes posible. Precisamente esta estrategia posibilita a los estudiantes de pedagogía asumir roles, desafíos y responsabilidades profesionales en contextos laborales, aprender el marco normativo de la profesión y poner a prueba su vocación y eficacia profesional. Cuando ésta se encuentra adecuadamente planificada y los estudiantes reciben el apoyo correspondiente, entonces desarrollan mayores niveles de IP (Madueño y Márquez, 2020). Por el contrario, cuando la práctica profesional no cumple estos requisitos, los estudiantes podrían desarrollar una menor IP o bien, una vez se insertan al campo laboral como docentes, se podría generar un choque de realidad, con el consecuente riesgo del abandono de la carrera profesional.

Una segunda categoría de hallazgos que desde los estudios revisados se desprende, son las características psicológicas de los futuros docentes. Corresponde a la dimensión personal involucrada en la construcción de la IP docente. Se encontró que, para desarrollar la IP, es necesario que los estudiantes cuenten con un conjunto de capacidades cognitivas, características de personalidad, experiencias educativas y personales 
de la infancia, además de las creencias acerca de la profesión. Destacan capacidades cognitivas y características de personalidad necesarias para lograr un aprendizaje profundo, significativo y reflexivo, que básicamente implica aprender a construir la propia IP. Algunos autores han destacado la necesidad de que los estudiantes sean protagonistas en la construcción de su IP, para lograr una adecuada identificación con la profesión (Beijaard, 2019) y una mayor conciencia acerca de lo que significa ser profesor(a). Probablemente estos hallazgos contribuyen a comprender mejor qué condiciones pueden favorecer o no el protagonismo de los estudiantes, en la construcción de su propia IP docente. Una dimensión psicológica particularmente importante son las experiencias infantiles ligadas a lo educativo, ya que a partir de éstas se va forjando la vocación docente, además de construir un conjunto de creencias o saberes subjetivos que permiten desarrollar una pre identidad docente, que es necesario de considerar en los procesos formativos para lograr la profesionalización docente.

Una tercera categoría incidente en el desarrollo de la IP docente durante la formación inicial, son los factores socioculturales. Para que el estudiante de pedagogía desarrolle una IP, es necesario que interactúe con los símbolos y representaciones sociales de la profesión, además de los grupos sociales relacionados con lo educativo (Hubbard, 2018). Es la integración de esta información y experiencias, la que permite al estudiante llegar a construir un significado subjetivo y personal de quién es como profesor(a) y qué es ser profesor(a). Un hallazgo obtenido en este estudio, que complementa lo anterior, es que para el desarrollo de la IP es preciso planear y sistematizar una adecuada inserción e interacción del futuro docente, con los grupos sociales que regulan, norman, educan y organizan la profesión docente. También es preciso integrar sistemáticamente en los procesos formativos, las representaciones sociales de la profesión docente, a fin de que el estudiante cuestione las normas, roles, estatus, valores y comportamientos con que la sociedad en general, identifica a los profesores(as).

Considerando que la IP no solo es un producto, sino que también un proceso, en este trabajo graficamos la forma en que los futuros docentes van construyendo su IP durante la formación inicial. Esta propuesta destaca un proceso de construcción conflictuado que se produce precisamente por la dificultad de integración de las dimensiones subjetivas y objetivas de la IP docente (Hendrikx, 2020; Živković, 2018). En la medida que el estudiante cuenta con los apoyos suficientes para lograr esta integración de saberes, es que persevera en la carrera profesional desarrollando altos niveles de identificación con la profesión. Desde esto, es urgente desarrollar competencias en los formadores, además de estrategias, disponer recursos y políticas en los programas de formación, que permitan abordar sistemáticamente la integración de estos saberes.

La IP como producto, desde los estudios revisados, consiste en el aprendizaje de (a) roles, (b) saberes, (c) tradiciones, (d) además del desarrollo de características de personalidad (e) y de un sentimiento de ser parte de la profesión docente. Cada uno de estos aprendizajes considera particularidades de lo que implica ser profesor(a), en función de la cultura. Sin embargo, en general se puede graficar a un docente como un profesional que tiene un rol educativo, social y moral altamente exigente (Le y Dwyer, 2019), para lo cual requiere dominar un extenso saber que va más allá de lo pedagógico y disciplinario y una personalidad fundamentalmente resiliente, que le permita trabajar bajo presión y con altos niveles de exigencia (Steenekamp et al., 2018). El docente es además un profesional que comparte con su gremio profesional y tiene la costumbre de educar en todo momento a sus estudiantes (Köksal, y Genç, 2019). Lo anterior, podría conllevar a que se sienta parte de la profesión o bien, a partir de estas elevadas exigencias, abandone la carrera profesional.

Finalmente, hemos presentado una categoría que da cuenta de las características metodológicas de los estudios revisados. Destaca la variedad de diseños e instrumentos de recolección de información utilizados. Lo anterior permite comprender de una manera más holista la dimensión subjetiva de la IP del docente. Sin embargo, futuros estudios deberían incluir mayormente diseños longitudinales, que permitan comprender el desarrollo de la IP desde una perspectiva temporal. También se requieren más estudios etnográficos y de investigación acción, no solo por la baja frecuencia de este enfoque en los estudios revisados, sino que fundamentalmente porque en un contexto socioeducativo altamente dinámico, es preciso identificar qué transformaciones sociales y culturales prescriben más o menos lo que significa ser docente en cada cultura. Por otro lado, también es necesario probar programas de desarrollo de la IP, que pongan como protagonistas del cambio a los principales actores de este proceso.

La profesionalización del profesor(a), en gran parte depende de cómo se identifica como docente. Formar a los futuros docentes hoy más que nunca implica desarrollar su IP. Ciertamente lograr un mayor conocimiento acerca de este proceso puede contribuir a la mejora de la formación docente y al desarrollo de un profesional mejor situado en el estatus de las profesiones. 


\section{CONCLUSIONES}

En base a la revisión de literatura realizada se puede concluir que el desarrollo de la IP del docente se ve influenciado por la formación inicial, las características psicológicas y los factores socioculturales. En el caso de la formación inicial, destaca el rol del: 1) programa de estudio, pues aquellos que se enfocan en integrar los conocimientos teórico prácticos, el aprendizaje autónomo, la reflexión, el trabajo en equipo, la nivelación de competencias y las actividades formativas, generan mayores niveles de desarrollo de la IP; 2) la figura del mentor y del académico, quienes pueden impulsar la identificación con la profesión o incluso promover el abandono de la carrera, por lo cual ambos deben ser agentes formativos que establezcan un vínculo de apoyo, confianza y respeto con los estudiantes, para que se produzca una construcción reflexiva de la IP; y 3) los procesos de práctica profesional, la cual debe encontrarse adecuadamente planificada, e iniciar lo antes posible, permitiendo a los estudiantes recibir el apoyo correspondiente, lo cual se relaciona con un mayor desarrollo de la IP. En cuanto a características psicológicas, se concluye que las capacidades cognitivas, características de personalidad, creencias acerca de la profesión, experiencias educativas y personales de la infancia, resultan fundamentales para desarrollar la IP. En lo que respecta a los factores socioculturales, se concluye que la planeación y sistematización de una adecuada inserción e interacción del estudiante, con grupos sociales que regulan, norman, educan y organizan la profesión docente, son factores necesarios para el desarrollo de la IP. Sumado a lo anterior, es necesario integrar sistemáticamente en los procesos formativos, las representaciones sociales de la profesión docente, permitiendo al estudiante cuestionar las normas, roles, estatus, valores y comportamientos con los cuales la sociedad en general, identifica a los profesores(as). Finalmente, se puede concluir, en base a los estudios revisados, que la IP como producto consiste en el aprendizaje de roles, saberes, tradiciones, además del desarrollo de características de personalidad y de un sentimiento de ser parte de la profesión docente, todo lo anterior en función de la cultura.

\section{AGRADECIMIENTOS}

Este trabajo es producto del proyecto Fondecyt Regular 1201084, financiado por la Agencia Nacional de Investigación y Desarrollo.

\section{REFERENCIAS}

Alvarado, M., Neira, M., y Westmacott, A., Collaborative reflective practice: its Influence on pre-service EFL teachers' emerging professional identities, https://doi.org/10.30466/IJLTR.2019.120736, Iranian Journal of Language Teaching Research, 7(3), 53-70 (2019)

Arnold, J., Artful praxis: experience, inquiry and consciousness, http://doi.org/10.1108/QRJ-05-2019-0041, Qualitative Research Journal, 19(4), 438-448 (2019)

Beijaard, D., Teacher learning as identity learning: models, practices, and topics,

https://doi.org/10.1080/13540602.2019.1542871, Teachers and Teaching, Theory and Practice, 25(1), 1-6 (2019)

Bolívar, A., Domingo, J., y Pérez-García, P., Crisis and reconstruction of teachers' professional identity: the case of secondary school teachers in Spain, https://doi.org/10.2174/1875399X01407010106, The Open Sports Science Journal, 7(Suppl-2, M4), 106-112 (2014)

Broaddus, K., From peacemaker to advocate: a preservice teacher's case study of an emergent reader, https://doi.org/10.1080/10862960009548096, Journal of Literacy Research, 32(4), 571-597 (2000)

Cardoso, I., Batista, P., y Graça, A., Professional identity in analysis: a systematic review of the literature, https://doi.org/10.2174/1875399X01407010083, The Open Sports Science Journal, 7(Suppl-2, M2), 83-97 (2014)

Carrier, S., Whitehead, A., y otros tres autores, The development of elementary teacher identities as teachers of science, https://doi.org/10.1080/09500693.2017.1351648, International Journal of Science Education, 39(13), 1733-1754 (2017)

Chikoko, R., y Msibi, T., The emerging professional teacher identity of early childhood and foundation phase pre-service teachers: implications for teacher education programmes, https://doi.org/10.17206/apjrece.2020.14.2.53, Asia-Pacific Journal of Research in Early Childhood Education, 14(2), 53-76 (2020).

Flores, L., Olave, I., y Villarreal, A., The impact of role modeling on the professional identity of pre-service teachers, https://doi.org/10.17509/ijal.v10i1.25024, Indonesian Journal of Applied Linguistics, 10(1), 143-152 (2020)

Hendrikx, W., What we should do vs what we do: teachers' professional identity in a context of managerial reform, https://doi.org/10.1080/03055698.2019.1620694, Educational Studies, 46(5), 607-623 (2020)

Hubbard, J., K-6 pre-service teachers' emerging professional identities as social studies educators, https://doi.org/10.1016/j.jssr.2018.04.002, The Journal of Social Studies Research, 43(3), 269-283 (2018)

Köksal, D., y Genç, G., Learning while teaching: student teachers' reflections on their teaching practicum, https://doi.org/10.17263/jls.631531, Journal of Language and Linguistic Studies, 15(3), 895-913 (2019)

Le, L.T., y Dwyer, L.G., Revisiting "Teacher as moral guide", in English language teacher education in contemporary Vietnam. Reforming vietnamese higher education by N. Nguyen y L. Tran, 245-267, Springer Vol. 50, Singapore (2019) 
Madueño, M., y Márquez, L., Formación de la identidad docente de estudiantes de la carrera de Educación Primaria desde la experiencia de la práctica profesional, http://dx.doi.org/10.4067/S0718-50062020000500057, Formación Universitaria, 13(5), 57-68 (2020)

McGee, E., When it comes to the mathematics experiences of black pre-service teachers... race matters, Teachers College Record,116(6), 1-30 (2014)

Nguyen, H., y Loughland, T., Pre-service teachers' construction of professional identity through peer collaboration during professional experience: a case study in Australia, https://doi.org/10.1080/10476210.2017.1353965, Teaching Education, 29(1), 81-97 (2018)

Rourke, A., y Snepvangers, K., Ecologies of practice in tertiary art and design: a review of two cases, http://dx.doi.org/10.1108/HESWBL-04-2015-0014, Higher Education, Skills and Work-Based Learning, 6(1), 69-85 (2016)

Sayago, Z., Chacón, M., y Rojas de Rojas, M., Construcción de la identidad profesional docente en estudiantes universitarios, ISSN: 1316-4910, Educere, 12(42), 551-561 (2008)

Steenekamp, K., van der Merwe, M., y Mehmedova, A.S., Enabling the development of student teacher professional identity through vicarious learning during an educational excursion, https://doi.org/10.15700/saje.v38n1a1407, South African Journal of Education, 38(1), 1-8 (2018)

Tezgiden, Y., A critical review of teacher education models, ISSN: 1307-3842, International Journal of Educational Policies, 10(2), 121-140 (2016)

van der Wal, M., Oolbekkink-Marchand, H., Schaap, H., y Meijer, P. Impact of early career teachers' professional identity tensions, https://doi.org/10.1016/j.tate.2019.01.001, Teaching and Teacher Education, 80, 59-70 (2019)

Zhu, G., Chinese student teachers' perspectives on becoming a teacher in the practicum: emotional and ethical dimensions of identity shaping, http://dx.doi.org/10.1080/02607476.2017.1341162, Journal of Education for Teaching, International Research and Pedagogy, 43(4), 491-495 (2017)

Živković, P., The dimensionality of student teacher professional identity, International Journal of Education Teacher, 8(15), 17-27 (2018) 